\title{
Effective treatment of alopecia universalis with oral tofacitinib: A case report
}

\author{
Lenah Shaikh, Amnah Almulhim, Manal Al Rabai, Yasir Shaikh
}

Dermatology Department, King Fahad Specialist hospital, Dammam, Saudi Arabia

Corresponding author: Dr. Lenah Shaikh, E-mail: Dr_lenah@hotmail.com

\begin{abstract}
Alopecia areata is a common autoimmune disease presenting itself with patches of hair loss on the scalp, eyebrows, eyelashes, or any part of the body. It may manifest itself as a single patch, involving the entire scalp (alopecia totalis), or affecting the entire body, thus the name alopecia universalis. Multiple lines of treatment may be employed, but no single most effective treatment exists, especially if the condition is generalized and, thus, becomes more difficult to treat. Herein, we report a case of alopecia universalis treated with oral tofacitinib with an excellent and persistent response one year after.
\end{abstract}

Key words: Alopecia areata; Alopecia universalis; Tofacitinib

\section{INTRODUCTION}

Alopecia areata (AA) is a common autoimmune disease with a lifetime incidence of $2 \%$ worldwide [1]. Its clinical presentation ranges from a single patch of nonscaring hair loss, the involvement of the entire scalp, also known as alopecia totalis (AT), or generalized hair loss involving the entire body, also known as alopecia universalis (AU) [2].

Its pathogenesis is a complex immune-mediated response with predominant CD8 T-cells leading to an attack on a hair follicle [3]. It has been documented as an increase in CD8/CD4 cells around a hair follicle in an area known as the bulb causing non-scaring hair loss $[4,5]$. An increase in substance p that mediates the transition of the hair cycle from anagen to catagen has been reported, thus resulting in hair fall [6].

There are several lines of treatment available for alopecia universalis, including topical and oral steroids, topical immunomodulators, but no FDA approved therapy as of yet. Recently, Janus kinase (JAKl-3) and tyrosine kinase TYK2 have been reported to be relevant [7]. JAK enzymes are implicated in a series of transcribing inflammatory proteins, which are involved in the pathogenesis of numerous autoimmune diseases, such as vitiligo, rheumatoid arthritis, atopic dermatitis, psoriasis/psoriatic arthritis, and alopecia areata. By blocking these enzymes, targeted treatment is achieved $[5,7,8]$.

Many cases are resistant to some, if not all, lines of treatment, especially long-standing cases. Thus, the possibility of a targeted treatment that is effective for cases such as alopecia universalis is promising.

\section{CASE REPORT}

We present the case of a 29-year-old female with a history of alopecia totalis present since 2015. It started with patchy hair loss limited to the scalp. The patient was previously seen in another hospital, where she was started on minoxidil $5 \%$ once daily initially with a good improvement for one year. She had been a known case of rheumatoid arthritis for ten years treated with methotrexate $22.5 \mathrm{mg}$ for one year, thereafter, in 2011, switched to rituximab at a dose of $1000 \mathrm{mg}$ once every six months. 
In 2017, the patient's alopecia relapsed in three months as alopecia universalis with hair loss on the scalp, eyebrows, eyelashes, and the entire body. At that time, she presented herself to our clinic. Other causes of alopecia were excluded by history taking and laboratory tests, including a thyroid function test with anti-thyroid antibodies, iron, vitamin $D$, vitamin $B_{12}$, which were all within normal limits.

In May 2018, after a discussion with the patient's rheumatologist, we decided to stop rituximab and, instead, switch her to tofacitinib $5 \mathrm{mg}$ orally twice daily, targeting both rheumatoid arthritis and alopecia universalis, in addition to topical minoxidil $5 \%$ once daily.

Baseline investigation was performed prior to starting tofacitinib, including complete blood count (CBC) with a differential to measure the amount of hemoglobin and the number of lymphocytes and neutrophils. In addition, the renal panel, liver panel, lipid profile, intradermal purified protein derivative (PPD) test, chest X-ray (CXR), hepatitis screening, and antinuclear antibodies (ANA) were all within normal limits. On each visit, vital signs were taken, a thorough physical examination was performed, and laboratory monitoring for $\mathrm{CBC}$ with a differential was conducted. All these were unremarkable.

An examination at presentation revealed diffuse hair loss on the scalp, eyelashes, eyebrows, and the entire body (Fig. la). On trichoscopy, follicular ostia were seen with black and yellow dots. No perifollicular erythema or scales were found. After three months of starting tofacitinib, fine patches of hair growth were observed on the scalp, eyebrows, and eyelashes (Fig. 1b). At six months, significant hair growth was noted on the eyebrows, eyelashes, and entire body, with the complete regrowth of the scalp hair (Figs. lc-d). At nine months, the length and density of the hair of the scalp and entire body increased (Figs. le-f). Eleven months after starting the treatment, the patient maintained excellent clinical improvement with an increasing hair length (Fig. $1 \mathrm{~g}$ ). At thirteen months, the patient maintained the hair growth all over the body, again with an increasing hair length (Figs. 1h-i). The patient has

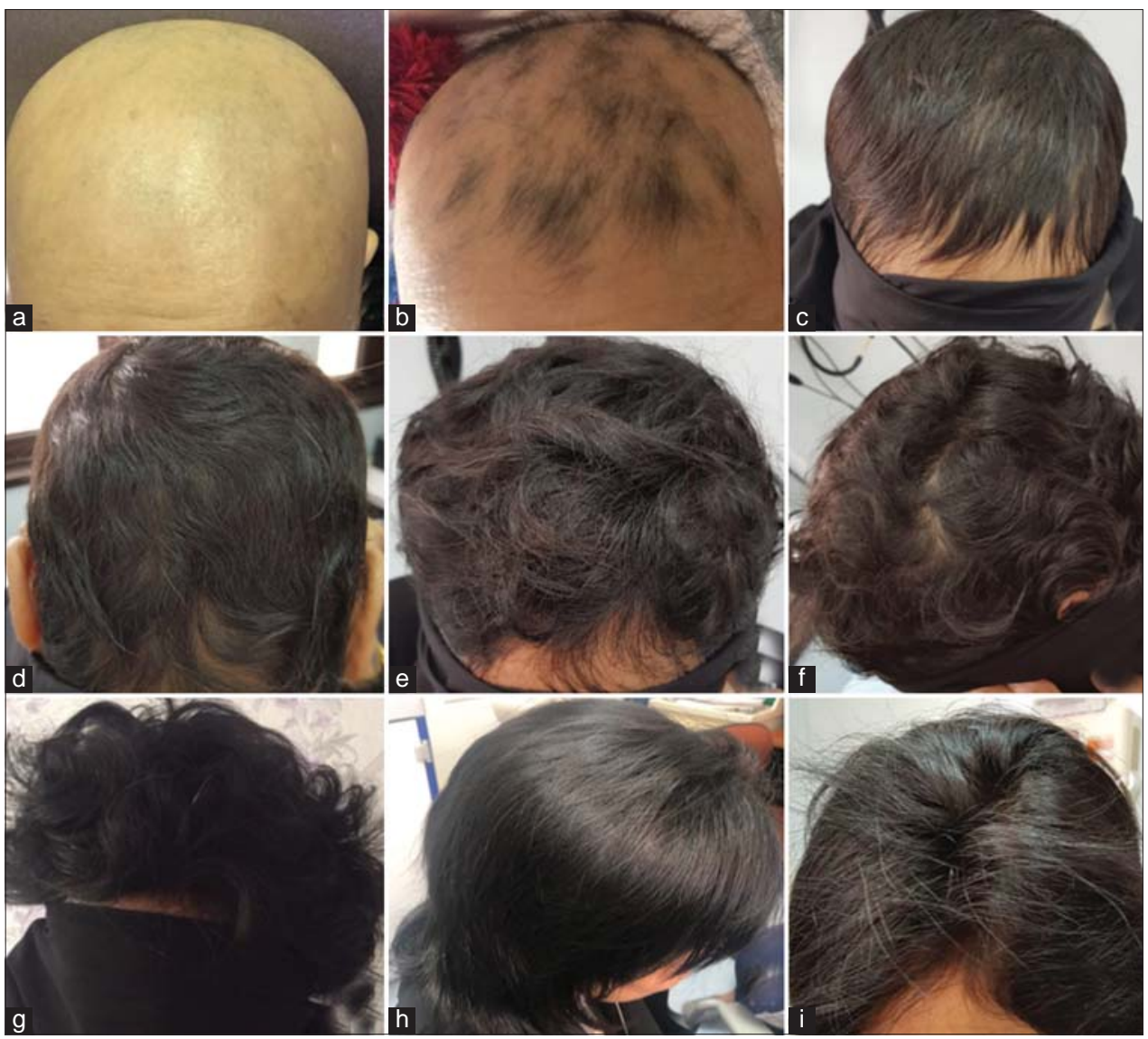

Figure 1: Clinical response to tofacitinib (a) at presentation, (b) after three months, (c-d) six months, (e-f) nine months, (g) eleven months, and $(\mathrm{h}-\mathrm{i})$ thirteen months after its first administration. 
stayed on tofacitinib for the last thirteen months with a good and sustainable response and without any side effects or relapses.

\section{DISCUSSION}

Alopecia areata (AA) is a known T-helper $1 \mathrm{~T}_{\mathrm{h}} \mathrm{l}$ disease involving cytokines such as interleukin-2 (IL-2), tumor necrosis factor alpha (TNF- $\alpha)$, and interferon gamma (IFN- $\gamma)$. The contribution of IFN- $\gamma$ leads to the release of a chain of cytokines IL-2, IL-7, IL-15, and IL-21, which are implicated in the signaling of the Janus kinase-STAT pathway [4,9]. JAK inhibitors interfere with the inflammatory process within the immune system, blocking a series of cytokine-mediated interactions in the JAK-STAT pathway. Tofacitinib is a targeted medication blocking JAKl-3 and has been shown in several studies to diminish or block the inflammatory cascade targeting the hair follicle in alopecia areata. Tofacitinib is now FDA approved for the treatment of rheumatoid arthritis, psoriatic arthritis, and ulcerative colitis $[4,8]$.

Our case report supports the results of prior reported cases. Crispin et al. published the results of a clinical trial that evaluated the efficacy of tofacitinib, and of the pan-JAK inhibitor, in the treatment of severe AA and its variants. These results suggest that tofacitinib is a safe and effective treatment for severe AA, AT, and AU [4]. Similar cases of alopecia universalis treated with tofacitinib were reported by Gupta [10]. Another case of alopecia universalis responding well to oral tofacitinib without significant side effects was reported by Hogan, who claimed that JAK inhibition may serve as an effective treatment modality for AA [11]. A comparable case reported by Ferreira et al. showed an excellent response to oral tofacitinib in an adolescent patient with alopecia areata [12].

Our patient obtained sustainable results after thirteen months of starting the treatment. Relapses were reported upon the discontinuation of the medication $[10,13,14]$. Relapses require longer followups in addition to changing the management plan with the treating rheumatologist.

This patient had two autoimmune diseases, probably with a similar pathogenesis. Thus, treating rheumatoid arthritis might result in treating alopecia as well. Nevertheless, the pathogenesis of alopecia areata is not always that clear. Therefore, treating other types of alopecia where it is the sole presentation in the patient is necessary to evaluate the effects of tofacitinib. Moreover, the patient was on minoxidil simultaneously with tofacitinib. Thus, the results might have been due to the synergistic effect of the medications. Nonetheless, minoxidil was not applied over the body, and so the effect is to be mainly attributed to tofacitinib.

Pictures of the face and body could not be taken due to the patient's refusal. Trichoscopy pictures were not taken for the same reason.

\section{CONCLUSION}

Our case report shows significant improvement of AU with oral tofacitinib with no reported side effects. However, further clinical trials are required in order to confirm the results with longer follow-ups while on and off medication.

\section{Consent}

The examination of the patient was conducted according to the principles of the Declaration of Helsinki. The authors certify that they have obtained all appropriate patient consent forms, in which the patients gave their consent for images and other clinical information to be included in the journal. The patients understand that their names and initials will not be published, and due effort will be made to conceal their identity, but that anonymity cannot be guaranteed.

\section{ACKNOWLEDGMENTS}

We would like to thank Dr. Faiza Al Jishi, consultant adult rheumatologist / KFSH-D, for contributing by providing tofacitinib to our patient.

\section{REFERENCES}

1. Villasante Fricke AC, Miteva M. Epidemiology and burden of alopecia areata: A systematic review. Clin Cosmet Investig Dermatol. 2015;8:397-403.

2. Hordinsky MK. Overview of alopecia areata. J Investig Dermatol Symp Proc. 2013;16:13-5.

3. Xing L, Dai Z, Jabbari A, Cerise JE, Higgins CA, Gong W, et al. Alopecia areata is driven by cytotoxic T lymphocytes and is reversed by JAK inhibition. Nat Med. 2014;20:1043-9.

4. Kennedy Crispin M, Ko JM, Craiglow BG, Li S, Shankar G, Urban JR, et al. Safety and efficacy of the JAK inhibitor tofacitinib citrate in patients with alopecia areata. JCI Insight. 2016;1:89776.

5. Park H, Yu DA, Kwon O. Janus kinase inhibitors: An innovative treatment for alopecia areata. J Dermatol. 2019;46:724-30.

6. Gilhar A, Kam Y, Assy B, Kalish RS. Alopecia areata induced in $\mathrm{C} 3 \mathrm{H} / \mathrm{HeJ}$ mice by interferon-gamma: Evidence for loss of immune privilege. J Invest Dermatol. 2005;124:288-9. 
7. Damsky W, King BA. JAK inhibitors in dermatology: The promise of a new drug class. J Am Acad Dermatol. 2017;76:736-44.

8. Shreberk-Hassidim R, Ramot Y, Zlotogorski A. Janus kinase inhibitors in dermatology: A systematic review. J Am Acad Dermatol. 2017;76:745-53.

9. Kasumagic-Halilovic E, Ovcina-Kurtovic N, Begovic B, Zecevic L. Interferon-gamma in patients with alopecia universalis. Our Dermatol Online. 2018;3: 229-32.

10. Gupta AK, Carviel JL, Abramovits W. Efficacy of tofacitinib in treatment of alopecia universalis in two patients. J Eur Acad Dermatol Venereol. 2016;30:1373-8.

11. Shivanna CB, Shenoy C, Priya RA. Tofacitinib (selective Janus kinase inhibitor 1 and 3): A promising therapy for the treatment of alopecia areata: A case report of six patients. Int J Trichology. 2018;10:103-7.

12. Berbert Ferreira R, Ferreira SB, Scheinberg SB. An excellent response to tofacitinib in a Brazilian adolescent patient with alopecia areata: A case report and a review of the literature. Clin Case Rep. 2019;7:2539-42.

13. Jabbari A, Sansaricq F, Cerise J, Chen JC, Bitterman A, Ulerio G, et al. An open-label pilot study to evaluate the efficacy of tofacitinib in moderate to severe patch-type alopecia areata, totalis, and universalis. J Invest Dermatol. 2018;138:1539-45.

14. Todberg T, Loft ND, Zachariae C. Improvement of psoriasis, psoriatic arthritis, and alopecia universalis during treatment with tofacitinib: A case report. Case Rep Dermatol. 2020;12:150-4.

Copyright by Shaikh, et al. This is an open access article distributed under the terms of the Creative Commons Attribution License, which permits unrestricted use, distribution, and reproduction in any medium, provided the original author and source are credited.

Source of Support: Nil, Conflict of Interest: None declared. 